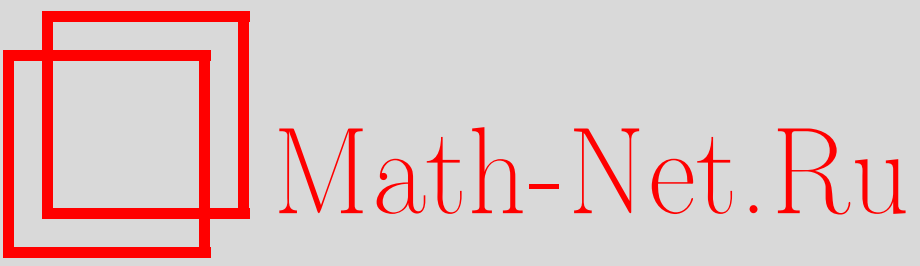

А. А. Дрокин, А. В. Шаповалов, И. В. Широков, Алгебра локальных симметрий уравнения Шредингера для атома водорода, ТМФ, 1996, том 106, номер 2, 273284

DOI: https://doi.org/10.4213/tmf1113

Использование Общероссийского математического портала Math-Net.Ru подразумевает, что вы прочитали и согласны с пользовательским соглашением http: //www.mathnet.ru/rus/agreement

Параметры загрузки:

IP : 52.6 .47 .48

26 апреля 2023 г., $10: 25: 47$ 
ТЕОРЕТИЧЕСКАЯ

И МАТЕМАТИЧЕСКАЯ

ФИЗИКА

Том 106, № 2

февраль, 1996

А. А. Дрокин, А.В. Шаповалов, И. В. Широков

\title{
АЛГЕБРА ЛОКАЛЬНЫХ СИММЕТРИЙ УРАВНЕНИЯ ШРЕДИНГЕРА ДЛЯ АТОМА ВОДОРОДА
}

\begin{abstract}
Дано полное описание всех локальных симметрий (представляющих собой дифференциальные операторы произвольного конечного порядка) стационарного уравнения Шредингера для атома водорода на основе редукции уравнения Шредингера для изотропного гармонического осциллятора к уравнению Шредингера для атома водорода, порождающей соответствующую редукцию алгебр симметрии. Показано, что все нетривиальные локальные операторы симетрии в случае $n$-мерного изотропного гармонического осциллятора принадлежат обертывающей алгебре $U(s u(n, C))$ алгебры $s u(n, C)$. В случае атома водорода все нетривиальные локальные симметрии составляют обертывающую алгебру $U(s o(4, C))$ алгебры $s o(4, C)$. Базис $s o(4, C)$ состоит из генераторов группы вращений и операторов Рунге-Ленца.
\end{abstract}

\section{1. ВВЕДЕНИЕ}

Локальные симметрии являются важным инструментом исследования групповых и алгебраических свойств линейных и нелинейных дифференциальных уравнений [1-4]. Множество локальных симметрий, коммутируюших с оператором уравнения на его решениях, образует бесконечномерную алгебру Ли $\mathcal{A}$, называемую алгеброй симметрии уравнения. Подалгебры алгебры $\mathcal{A}$ используются для построения точных решений дифференциальных уравнений. Например, теория разделения переменных строится на основе коммутативных подалгебр специального вида алгебры $\mathcal{A}$, порядок элементов которых не превышает порядка уравнения $[5,6]$. Некоммутативные подалгебры алгебры $\mathcal{A}$ использованы в работе [7] для интегрирования линейных дифференциальных уравнений в частных производных. Однако свойства алгебры $\mathcal{A}$ в целом для заданного уравнения изучены мало, т.к. полное описание алгебры симметрии известно лишь для очень ограниченного числа уравнений.

Прямое вычисление алгебры $\mathcal{A}$ для конкретного уравнения представляет собой сложную задачу. В многомерном случае алгебра $\mathcal{A}$ найдена таким способом для свободного уравнения Клейна-Гордона в пространстве Минковского $R_{1,3}$ в [8], для волнового уравнения - в [9]. В работе [10] алгебра $\mathcal{A}$ найдена для уравнения Лапласа-Бельтрами в пространстве $R_{p, q}$ с помощью теорем о структуре алгебры $\mathcal{A}$, минуя прямое ее вычисление. Там же доказана теорема о том, что все локальные симметрии линейных уравнений с числом независимых переменных больше двух линейны.

В данной работе вычисляется алгебра $\mathcal{A}_{H}$ всех локальных симметрий уравнения 
Шредингера для атома водорода, коммутируюших с оператором уравнения $H$. Последний имеет переменные коэффициенты, что отличает данную задачу от исследованных ранее случаев.

Для стационарного уравнения Шредингера алгебра $\mathcal{A}$ симметрий, коммутируюших с оператором уравнения на его решениях, отличается от алгебры $\mathcal{A}_{H}$ операторов симметрии, коммутируюших с $H$ на всей области определения $H$, идеалом, состояшим из тривиальных операторов вида $R H$, где $R$ - произвольньй линейный оператор. Это обстоятельство следует из обших теорем работ $[2,3,11]$ и позволяет без потери обшности ограничиться рассмотрением алгебры $\mathcal{A}_{H}$.

Изучение симметрии уравнения Шредингера для атома водорода является одной из классических задач математической физики. Фок [12] и Баргман [13] установили инвариантность уравнения Шредингера в импульсном представлении относительно группы $O(4)$. Дальнейшее развитие эти идеи получили в работах [14-18], где была развита концепция динамической симметрии (алгебры, генерируюшей спектр). Операторы алгебры динамической симметрии принадлежат алгебре $\mathcal{A}$. Базис пространства решений в некоторых случаях может быть построен действием специально подобранных операторов динамической симметрии на некоторое единственное исходное решение. Более полную информацию можно найти в обзорах и монографиях [19-22]. Отметим также, что известные уравнения, для которых решения построены с помощью динамических симметрий [20-22], допускают интегрирование методом разделения переменньх, т.е. с помошью коммутативных подалгебр алгебры $\mathcal{A}$. Найденная Фоком и Баргманом $O(4)$-симметрия уравнения Шредингера для атома водорода содержится в алгебре $\mathcal{A}_{H}$. В координатном представлении часть генераторов группы $O(4)$ становятся дифференциальными операторами второго порядка (вектор Рунге-Ленца (см., например, [22])) и являются элементами алгебры $\mathcal{A}_{H}$. В импульсном представлении операторы, образующие конечномерную подалгебру $s o(4)$ алгебры $\mathcal{A}_{H}$, реализуются дифференциальными операторами первого порядка и генерируют группу $S O(4)$.

Полная алгебра $\mathcal{A}_{H}$ (т.е. дифференциальные операторы симметрии произвольного порядка уравнения Шредингера с кулоновским полем, не зависящие от энергии как от параметра уравнения) оставалась ненайденной. Нахождение алгебры $\mathcal{A}_{H}$ может быть полезно с различных точек зрения. В частности, представляет интерес выяснить, сушествуют ли операторы симметрии высокого порядка, не разложимые в произведение операторов симметрии первого порядка или в произведение операторов первого порядка и компонент вектора Рунге-Ленца. Такие операторы можно было бы рассматривать как аналоги вектора Рунге-Ленца высокого порядка.

Для нахождения алгебры $\mathcal{A}_{H}$ в основу нашего подхода положена известная редукция уравнения Шредингера для четырехмерного изотропного гармонического осциллятора к уравнению Шредингера для атома водорода [23]. Применение метода редукции для исследования алгебры симметрии редуцированного уравнения в обшем случае сводится к решению следуюших задач:

1) найти все локальные симметрии исходного уравнения;

2) выяснить связь симметрии исходного и редуцированного уравнений, в частности, установить, является ли алгебра симметрии редуцированного уравнения некоторым подмножеством алгебры симметрии исходного уравнения (это подмножество будем называть редукцией алгебры симметрии исходного уравнения); 
3) найти в явном виде алгебру симметрии редуцированного уравнения.

Как правило, алгебра симметрии исходного уравнения устроена достаточно просто: в большинстве случаев это обертываюшая алгебра некоторой алгебры Ли. Вторая задача нетривиальна, поскольку алгебра симметрии редуцированного уравнения может быть как у́же, так и шире алгебры симметрии исходного уравнения. В качестве примера рассмотрим уравнение

$$
\Delta_{1,2} \psi \equiv\left(\partial_{t}^{2}-\partial_{x}^{2}-\partial_{y}^{2}\right) \psi=0 .
$$

Редукция этого уравнения по оператору $Y=\partial_{y}$ подразумевает ограничение его решений дополнительным условием $Y \psi=0$, что приводит к (редуцированному) уравнению

$$
H_{r} \psi \equiv\left(\partial_{t}^{2}-\partial_{x}^{2}\right) \psi=0 \longleftrightarrow\left\{\Delta_{1,2} \psi=0, \quad Y \psi=0\right\} .
$$

Алгеброй Ли операторов симметрии первого порядка исходного уравнения (1.1) является конформная алгебра $k_{1,2}$. Аналогичная алгебра Ли редуцированного уравнения (1.2) бесконечномерна. Третья задача в обшем случае также нетривиальна. В настоящей работе предъявлен алгоритм решения этой проблемы в случае, когда алгебра симметрии исходного уравнения принадлежит обертывающей алгебре некоторой полупростой алгебры Ли, а оператор $Y$, по которому производится редукция, принадлежит подалгебре Картана.

Рассмотрим кратко, следуя [23], переход от уравнения Шредингера для гармонического осцилятора к уравнению Шредингера для атома водорода.

Стационарное уравнение Шредингера для четырехмерного изотропного гармонического осциллятора запишем в виде

$$
H \psi(x)=\frac{1}{2} \sum_{j=1}^{4}\left(-\partial_{x_{j}}^{2}+\omega^{2} x_{j}^{2}\right) \psi(x)=\varepsilon \psi(x) .
$$

Уравнение (1.3) в системе координат

$$
\begin{aligned}
x_{1} & =\sqrt{r} \cos \frac{\theta}{2} \cos \frac{\varphi+\alpha}{2}, & x_{2} & =\sqrt{r} \sin \frac{\theta}{2} \cos \frac{\varphi-\alpha}{2}, \\
x_{3} & =\sqrt{r} \cos \frac{\theta}{2} \sin \frac{\varphi+\alpha}{2}, & x_{4} & =\sqrt{r} \sin \frac{\theta}{2} \sin \frac{\varphi-\alpha}{2}
\end{aligned}
$$

принимает вид

$$
\begin{aligned}
& -2\left\{\frac{1}{r} \frac{\partial}{\partial r}\left(r^{2} \frac{\partial}{\partial r}\right)+\frac{1}{r}\left[\frac{1}{\sin \theta} \frac{\partial}{\partial \theta}\left(\sin \theta \frac{\partial}{\partial \theta}\right)+\right.\right. \\
& \left.\left.+\frac{1}{\sin ^{2} \theta}\left(\frac{\partial^{2}}{\partial \varphi^{2}}+\frac{\partial^{2}}{\partial \alpha^{2}}-2 \cos \theta \frac{\partial^{2}}{\partial \varphi \partial \alpha}\right)\right]-\frac{\omega^{2} r}{4}\right\} \psi=\varepsilon \psi .
\end{aligned}
$$

Решение этого уравнения, не зависяшее от переменной $\alpha$, совпадает с решением уравнения Шредингера для атома водорода

$$
\begin{aligned}
H_{r} \psi=\{ & -\frac{1}{2}\left[\frac{1}{r^{2}} \frac{\partial}{\partial r}\left(r^{2} \frac{\partial}{\partial r}\right)+\right. \\
& \left.\left.+\frac{1}{r^{2}}\left[\frac{1}{\sin \theta} \frac{\partial}{\partial \theta}\left(\sin \theta \frac{\partial}{\partial \theta}\right)+\frac{1}{\sin ^{2} \theta} \frac{\partial^{2}}{\partial \varphi^{2}}\right]\right]-\frac{e}{r}\right\} \psi=E \psi
\end{aligned}
$$


где $e=-\frac{\varepsilon}{4}, E=-\frac{\omega^{2}}{8}$.

Этому переходу можно придать алгебраический вид, в котором множество решений уравнения (1.3) редуцируется по оператору симметрии $Y=\partial_{\alpha}$, именно:

$$
\operatorname{Ker}\left(H_{r}-E\right)=\operatorname{Ker}(H-\varepsilon) \cap \operatorname{Ker} Y .
$$

В частности, уравнение (1.5) эквивалентно системе

$$
H \psi=\varepsilon \psi, \quad Y \psi=0
$$

В координатах $(x)$ оператор $Y$ имеет вид $Y=\frac{1}{2}\left(L_{13}-L_{24}\right)$, где $L_{k j}=x_{k} \partial_{x_{j}}-x_{j} \partial_{x_{k}}$.

В таком подходе удобно исследовать алгебраические свойства редуцированных уравнений, наследующих часть свойств исходного уравнения.

Следуя изложенной выше схеме, рассмотрим алгебру симметрии уравнения Шредингера для гармонического осциллятора.

\section{2. АЛГЕБРА СИММЕТРИИ КВАНТОВОГО $n$-МЕРНОГО ИЗОТРОПНОГО ГАРМОНИЧЕСКОГО ОСЦИЛЛЯТОРА}

С алгебраической точки зрения четырехмерный изотропный гармонический осциллятор принципиально не отличается от $n$-мерного, поэтому исследование симметрии уравнения Шредингера для изотропного гармонического осциллятора и редуцированного из него уравнения по некоторому оператору симметрии $Y$ мы проведем для $n$-мерного случая.

Рассмотрим $n$-мерное стационарное уравнение Шредингера с потенциалом изотропного гармонического осциллятора

$$
H \psi \equiv \frac{1}{2} \sum_{j=1}^{n}\left(-\partial_{x_{j}}^{2}+\omega^{2} x_{j}^{2}\right) \psi=\varepsilon \psi, \quad \omega>0 .
$$

Введем интегральное преобразование Вейерштрасса [24]

$$
\psi(x)=\int_{-\infty}^{+\infty} K(x, z) \varphi(z) d z
$$

с ядром $K(x, z)=\exp \left(-\omega z^{2}+2 i \omega(x \cdot z)+\omega x^{2} / 2\right)$ и обратное преобразование

$$
\varphi(z)=\int_{-\infty}^{+\infty} \bar{K}(x, z) \psi(x) d x
$$

с ядром $\bar{K}(x, z)=\left(\frac{\omega}{\pi}\right)^{n} \exp \left(\omega z^{2}-2 i \omega(x \cdot z)-\omega x^{2} / 2\right), x, z \in R^{n}$. Формулы обрашения принимают вид

$$
\begin{aligned}
& \int_{-\infty}^{+\infty} K(x, z) \bar{K}\left(x^{\prime}, z\right) d z=\delta\left(x-x^{\prime}\right), \\
& \int_{-\infty}^{+\infty} K(x, z) \bar{K}\left(x, z^{\prime}\right) d x=\delta\left(z-z^{\prime}\right) .
\end{aligned}
$$


Обозначим через $\Omega$ и $\bar{\Omega}$ функциональные пространства образов и прообразов преобразования Вейерштрасса $(2.2)$ и (2.3), соответственно,т.е. $\psi(x) \in \Omega, \varphi(z) \in \bar{\Omega}$.

Отметим, что функции $K(x, z), \bar{K}(x, z)$ обладают свойствами

$$
\begin{aligned}
& \left(\partial_{x_{j}}-\omega x_{j}\right) K(x, z)=2 i \omega z_{j} K(x, z), \\
& \left(\partial_{x_{j}}+\omega x_{j}\right) K(x, z)=-i \partial_{z_{j}} K(x, z), \\
& \left(\partial_{x_{j}}+\omega x_{j}\right) \bar{K}(x, z)=-2 i \omega z_{j} \bar{K}(x, z), \\
& \left(\partial_{x_{j}}-\omega x_{j}\right) \bar{K}(x, z)=-i \partial_{z_{j}} \bar{K}(x, z) .
\end{aligned}
$$

Если функции $\psi, \varphi$ связаны соотношением $(2.2): \psi(x) \longleftrightarrow \varphi(z)$, то

$$
\begin{aligned}
& \left(\partial_{x_{j}}-\omega x_{j}\right) \psi(x) \longleftrightarrow 2 i \omega z_{j} \varphi(z), \\
& \left(\partial_{x_{j}}+\omega x_{j}\right) \psi(x) \longleftrightarrow i \partial_{z_{j}} \varphi(z) .
\end{aligned}
$$

Отсюда непосредственно следует, что преобразования (2.2), (2.3) осуществляют не только взаимнооднозначные отображения пространств, $\Omega \longleftrightarrow \bar{\Omega}$, но и операторов, действуюших в этих пространствах. Именно, каждому линейному дифференциальному оператору $X\left(x, \partial_{x}\right)$ с аналитическими коэффициентами, действующему в пространстве $\Omega$, взаимнооднозначно соответствует аналогичный оператор $\bar{X}\left(z, \partial_{z}\right)$, действующий в пространстве $\bar{\Omega}: X\left(x, \partial_{x}\right) \longleftrightarrow \bar{X}\left(z, \partial_{z}\right)$. Очевидно также, что это отображение является изоморфизмом ассоциативных алгебр аналитических линейных дифференциальных операторов, действуюших в пространствах $\Omega, \bar{\Omega}$.

При отображении (2.3) уравнение (2.1) перейдет в уравнение

$$
\bar{H} \varphi(z) \equiv \omega\left(\sum_{j} z_{j} \partial_{z_{j}}+\frac{n}{2}\right) \varphi(z)=\varepsilon \varphi(z) .
$$

Согласно общим теоремам работ $[2,3,11]$ нетривиальные операторы симметрии уравнения (2.1) следует искать в классе линейных дифференциальных операторов $X$, не зависящих от параметра $\varepsilon$ и определяемых уравнением

$$
[H, X]=0 .
$$

Множество таких операторов образует алгебру Ли относительно коммутатора линейных операторов, которую мы будем рассматривать в качестве алгебры симметрии $\mathcal{A}$ уравнения (2.4). В таком подходе исключаются из рассмотрения тривиальные операторы симметрии, переводящие всякое решение уравнения (2.4) в нулевое.

При отображении (2.3) уравнение (2.5) перейдет в уравнение

$$
[\bar{H}, \bar{X}]=0 .
$$

Оператор $\bar{H}$ является оператором растяжений по координатам $z_{j}$, поэтому нетрудно непосредственно найти обшее решение уравнения (2.6) в классе дифференциальных операторов. Оно имеет вид $\bar{X}=\bar{X}\left(C_{j k}\right)$, здесь $\bar{X}\left(C_{j k}\right)$ - произвольный полином от операторов $C_{j k}=z_{j} \partial_{z_{k}}$. Введем обозначения $\bar{X}_{j k}=-\omega\left(C_{j k}+C_{k j}\right) ; \bar{L}_{j k}=C_{j k}-C_{k j}$. Совершая обратный переход (2.2), получим, что обшим решением уравнения (2.5) в классе 
дифференциальных операторов является произвольная аналитическая функция от операторов $X_{j k}, L_{j k}$. Последние имеют вид

$$
X_{j k}=\partial_{x_{j} x_{k}}-\omega^{2} x_{j} x_{k}, \quad L_{j k}=x_{j} \partial_{x_{k}}-x_{k} \partial_{x_{j}}
$$

и образуют алгебру Ли $u(n, C)$ относительно обычного коммутатора линейных операторов. Если исключить из этой алгебры тривиальньй оператор $H=-\frac{1}{2} \sum_{j} X_{j j}$, то остальные операторы будут составлять алгебру $s u(n, C)$. Таким образом, справедлива

ТЕОРема 2.1. Все нетривиальные дифференциальные операторы симметрии стационарного уравнения Шредингера с потенииалом $n$-мерного изотропного гармонического осииллятора (2.1) принадлежсат обертывающей алгебре $U(s u(n, C))$ алгебры $s и(n, C)$, которая имеет базис (2.7).

\section{3. АЛГЕБРА СИММЕТРИИ УРАВНЕНИЯ ШРЕДИНГЕРА ДЛЯ АТОМА ВОДОРОДА}

Проведем редукцию уравнения (2.1) по некоторому оператору $Y \in s u(n, C)$, полагая

$$
H_{r} \psi=\varepsilon \psi \Longleftrightarrow\{H \psi=\varepsilon \psi, \quad Y \psi=0\} .
$$

Нетривиальные операторы симметрии $X$ системы (3.1) определяются уравнениями

$$
\begin{aligned}
{[H, X] } & =Q Y, \\
{[Y, X] } & =R Y .
\end{aligned}
$$

Рассмотрим уравнение $(3.2)$ в пространстве $\bar{\Omega}$ :

$$
[\bar{H}, \bar{X}]=\overline{Q Y} \text {. }
$$

Перейдем в уравнении (3.4) к сферической системе координат: $\left(z_{1}, \ldots, z_{n}\right) \rightarrow(\rho$, $\left.\theta_{1}, \ldots, \theta_{n-1}\right)$. Здесь $\theta_{j}$ - угловые переменные, $\rho^{2}=\sum_{j=1}^{n} z_{j}^{2}$. В этих координатах $\bar{H}=\omega\left(\rho \partial_{\rho}+n / 2\right)$ и (3.4) имеет вид

$$
[\bar{H}, \bar{X}]=\omega \rho \frac{\partial \bar{X}}{\partial \rho}=\overline{Q Y}
$$

Решение последнего уравнения можно представить в виде

$$
\bar{X}=\bar{X}_{0}\left(\theta, \partial_{\theta}\right)+\overline{B Y}
$$

где

$$
\left[\bar{H}, \bar{X}_{0}\right]=0, \quad \bar{B}=\int_{\rho_{0}}^{\rho} \frac{\bar{Q} d \rho}{\omega \rho} .
$$

Содержательную информацию несет оператор $\bar{X}_{0}$, поэтому, не теряя общности, можно положить $\bar{B}=\bar{Q}=0$. 
ЗАмЕчАниЕ. К оператору $\bar{X}$ можно добавить тривиальное решение $R\left(\theta, \partial_{\theta}\right) f(\bar{H})$, где $f(\bar{H})$ - полином по $\bar{H}$. На решениях системы (3.1) этот оператор примет вид $R\left(\theta, \partial_{\theta}\right) f(\varepsilon)$ и его можно включить в первый член правой части (3.5). Поэтому оператор $R\left(\theta, \partial_{\theta}\right) f(\bar{H})$ в (3.5) опушен. Отсюда следует, что для любого решения $X$ уравнения (3.2) существует такой оператор $B$, что $X=X_{0}+B Y$, где $\left[H, X_{0}\right]=0$. Таким образом, не теряя обшности, можно положить в (3.2) $Q=0$.

Аналогично можно показать, что $R=0$ в (3.3). В системе координат, где $\bar{Y}=\partial_{y}$, уравнение (3.3) имеет вид $\partial \bar{X} / \partial y=\overline{R Y}$. Его решение можно представить в виде $\bar{X}=$ $\bar{X}_{0}+\overline{A Y}$, где $\partial \bar{X}_{0} / \partial y=0, \bar{A}=\int_{0}^{y} \bar{R} d y$. Повторяя вышеприведенные рассуждения, положим $\bar{R}=0$.

Таким образом, справедлива

Теорема 3.1. Все нетривиальные дифференциальные операторы симметрии $X$ редуцированного уравнения (3.1) определяются уравнениями

$$
[H, X]=[Y, X]=0 .
$$

Из теоремы 3.1 непосредственно получаем

СлЕДСтвИЕ 3.1. Алгебра $\mathcal{A}_{H}$ локальных симметрий уравнения Шредингера (1.5) для атома водорода является редукиией алгебры $U(s u(4, C))$ с помощью опеpamopa

$$
Y=\frac{1}{2}\left(L_{13}-L_{24}\right)
$$

именно:

$$
\mathcal{A}_{H}=\{X \in U(s u(4, C)) \mid[Y, X]=0\} .
$$

Перейдем к нахождению алгебры симметрии редуцированного уравнения в явном виде. В нашем случае оператор $Y$ вида (3.6) принадлежит подалгебре Картана полупростой алгебры $s u(4, C)$, что является существенным упрощением задачи.

Предварительно расмотрим более общую задачу редукции обертывающей алгебры $U(L)$ полупростой алгебры $L$ по элементу $Y$ из подалгебры Картана. Другими словами, требуется найти подалгебру $U_{Y}(L)$ алгебры $U(L)$, элементы которой коммутируют с оператором $Y$ :

$$
U_{Y}(L)=\{X \in U(L) \mid[X, Y]=0\} .
$$

Выберем в алгебре $L$ базис так, чтобы матрица $\operatorname{ad} Y$ была диагональной:

$$
\operatorname{ad} Y=\operatorname{diag}\left(\lambda_{1}, \ldots, \lambda_{1}, \ldots, \lambda_{t}, \ldots, \lambda_{t}, 0, \ldots, 0\right),
$$

где $\lambda_{1} \neq \lambda_{2} \neq \cdots \neq \lambda_{t}$ - характеристические числа матрицы ad $Y$ в пространстве $L$. Разложим $L$ в прямую сумму корневых подпространств

$$
L=\bigoplus_{i=1}^{t} E_{i} \oplus E_{0}
$$


Здесь $E_{i}=\left\{X \in L \mid[Y, X]=\lambda_{i} X\right\}$ - корневое пространство, соответствуюшее корню $\lambda_{i}, \operatorname{dim} E_{i}=s\left(\lambda_{i}\right)$ - кратность корня. Подпространство $E_{0}$, отвечаюшее нулевому характеристическому числу, имеет размерность

$$
\operatorname{dim} E_{0}=\operatorname{dim} L-\sum_{i=1}^{t} s\left(\lambda_{i}\right)
$$

и является подалгеброй алгебры $L$ вследствие тождества Якоби. Очевидно, что

$$
E_{0} \subset U_{Y}(L) \Rightarrow U\left(E_{0}\right) \subset U_{Y}(L),
$$

т.к. $E_{0}$ состоит из элементов алгебры $L$, коммутируюших с $Y$.

Рассмотрим структуру обертывающей алгебры $U(L)$, возникаюшую вследствие корневого разложения (3.7). Введем пространства

$$
U_{\vec{n}}=E_{1}^{n_{1}} \otimes \cdots \otimes E_{t}^{n_{t}}, \quad n_{i} \geq 0, \quad \vec{n}=\left(n_{1}, \ldots, n_{t}\right), \quad n=\sum_{i=1}^{t} n_{i}
$$

с базисом в виде упорядоченных мономов

$$
\left\{X_{\left(a_{1}^{1}\right.}^{1} \ldots X_{\left.a_{n_{1}}^{1}\right)}^{1} \ldots X_{\left(a_{1}^{t}\right.}^{t} \ldots X_{\left.a_{n_{t}}^{t}\right)}^{t}\right\}
$$

где $\left\{X_{a^{i}}^{i}\right\}$ - базис пространства $E_{i}, a^{i}=1, \ldots, s\left(\lambda_{i}\right)=\operatorname{dim} E_{i}$. Круглые скобки означают симметризацию. Определим пространство

$$
U^{(r)}=\bigoplus_{\substack{n_{1}+n_{2}+\cdots+n_{t}=n \\ n=0,1, \ldots, r}} U_{\vec{n}} \otimes E_{0}^{r-n}
$$

как линейную оболочку всевозможных мономов порядка $r$, причем имеет место разложение $U(L)=\bigoplus_{r=0} U^{(r)}$. Действие ad $Y$ естественно продолжается в обертываюшую алгебру $U(L)$ :

$$
\forall X \in U(L) \quad \operatorname{ad} Y(X)=[Y, X]
$$

Пространства $U^{(r)}$ инвариантны относительно действия оператора ad $Y$ на $U(L)$. Очевидно, что

$$
\operatorname{ad} Y\left(U_{\vec{n}}\right)=\left(\sum_{i=1}^{t} n_{i} \lambda_{i}\right) U_{\vec{n}}
$$

т.е. все элементы пространства $U_{\vec{n}}$ являются собственными для оператора ad $Y$ с одним и тем же собственным значением $\sum_{i=1}^{t} n_{i} \lambda_{i}$.

ОПРЕДЕЛЕНИЕ 3.1. Число $\sum_{i=1}^{t} n_{i} \lambda_{i}$ назовем весом пространства $U_{\vec{n}}$.

Обозначим через $W_{\vec{n}}$ пространство нулевого веса, т.е. пространства $U_{\vec{n}}$, для которого

$$
\sum_{i=1}^{t} n_{i} \lambda_{i}=0 .
$$

Из вышеприведенных рассуждений следует 
Теорема 3.2. Редущированная алгебра $U_{Y}(L)$ представляется в виде

$$
U_{Y}(L)=\bigoplus_{r=0}^{\infty}\left(\bigoplus_{\substack{n_{1}+n_{2}+\cdots+n_{t}=n \\ n=0,1, \ldots, r}} W_{\vec{n}} \otimes E_{0}^{r-n}\right)
$$

(здесь принято соглашение $\left.W_{\vec{n}} \otimes E_{0}^{r-n}\right|_{n=0}=E_{0}^{r}$ ).

Поскольку имеет место однозначное соответствие

$$
W_{\vec{n}} \longleftrightarrow \vec{n}=\left(n_{1}, \ldots, n_{t}\right)
$$

то решение задачи сводится к нахождению всех целочисленных положительных векторов $\vec{n}$, удовлетворяющих условию (3.8). Произведению пространств $W_{\vec{n}} \otimes W_{\vec{m}}$ отвечает вектор $\vec{n}+\vec{m}=\left(n_{1}+m_{1}, \ldots, n_{t}+m_{t}\right)$.

Множество решений уравнения (3.8) имеет конечное число частных решений - образуюших так, что все решения являются линейными комбинациями образуюших с положительными целочисленными коэффициентами. Другими словами, алгебра $U_{Y}(L)$ порождена конечным множеством операторов, построенных по системе образующих. Именно, пусть известны $\vec{n}_{1}, \ldots, \vec{n}_{\alpha}$ - положительные целочисленные векторы, порождаюшие все решения уравнения (3.8), т.е. для любого решения уравнения (3.8) $\vec{m}$ существуют такие положительные целые числа $\mu_{1}, \ldots, \mu_{\alpha}$, что $\vec{m}=\sum_{i=p}^{\alpha} \mu_{p} \vec{n}_{p}$. Пусть $\left\{Z_{a}^{p}\right\}$ - базис пространства $W_{\vec{n}_{p}}, a=1, \ldots, \operatorname{dim} W_{\vec{n}_{p}},\left\{Y_{b}\right\}$ - базис подалгебры $E_{0}$, $b=1, \ldots, \operatorname{dim} E_{0}$. Очевидно, что любой элемент алгебры $U_{Y}(L)$ состоит из линейных комбинаций произведений операторов $Y_{b}, Z_{a}^{p}$, и в этом смысле алгебра $U_{Y}(L)$ порождается этими операторами.

Применим изложенный выше алгоритм для нахождения локальных симметрий уравнения Шредингера для атома водорода. Выберем в алгебре $s u(4, C)$ базис, в котором оператор ad $Y$ диагонален. Разложение (3.7) имеет вид

$$
s u(4, C)=E_{1} \oplus E_{2} \oplus E_{0} ; \quad \lambda_{1}=i, \quad \lambda_{2}=-i .
$$

Алгебра $E_{0}$ состоит из прямой суммы одномерной алгебры $\{Y\}$ и алгебры $s o(4, C)$ : $E_{0}=\{Y\} \oplus s o(4, C)$. Алгебра $s o(4, C)$ является алгеброй Ли нетривиальных симметрий уравнения $(1.5)$, и ее базис состоит из трех операторов врашений $\tilde{L}_{i k}$ в трехмерном пространстве и трех компонент $R_{i}$ вектора Рунге-Ленца:

$$
\begin{aligned}
\tilde{L}_{12} & =\frac{1}{2}\left(L_{13}+L_{24}\right), & \tilde{L}_{13} & =\frac{1}{2}\left(L_{34}-L_{12}\right), \\
R_{1} & =\frac{1}{4}\left(X_{34}-X_{12}\right), & \tilde{L}_{23} & =\frac{1}{2}\left(L_{23}-L_{14}\right) ; \\
R_{2} & =-\frac{1}{4}\left(X_{14}+X_{23}\right), & R_{3} & =\frac{1}{8}\left(X_{22}+X_{44}-X_{11}-X_{33}\right) .
\end{aligned}
$$

Здесь обозначено

$$
\tilde{L}_{i k}=q_{i} \partial_{q_{k}}-q_{k} \partial_{q_{i}} ; \quad R_{i}=\sum_{k \neq i} \tilde{L}_{i k} \partial_{q_{k}}-\partial_{q_{i}}+\frac{e q_{i}}{r} ;
$$

$\left(q_{1}, q_{2}, q_{3}\right)$ - декартовы координаты: $q_{1}=r \sin \theta \cos \varphi, q_{2}=r \sin \theta \sin \varphi, q_{3}=r \cos \theta$. 
Корневое пространство $E_{1}$ имеет базис, состоящий из векторов $G_{1}, G_{3}, G_{5}, G_{7}$ : $\left[Y, G_{1,3,5,7}\right]=i G_{1,3,5,7}$, где

$$
\begin{aligned}
G_{1} & =\frac{1}{2}\left(L_{14}+L_{23}\right)+\frac{i}{2}\left(L_{12}+L_{34}\right), & G_{3} & =\frac{1}{2}\left(X_{14}-X_{23}\right)+\frac{i}{2}\left(X_{12}+X_{34}\right), \\
G_{5} & =\frac{1}{2}\left(X_{11}-X_{33}\right)+i X_{13}, & G_{7} & =X_{24}+\frac{i}{2}\left(X_{22}-X_{44}\right) .
\end{aligned}
$$

Векторы $G_{2}, G_{4}, G_{6}, G_{8}$ составляют базис пространства $E_{2}:\left[Y, G_{2,4,6,8}\right]=-i G_{2,4,6,8}$, где

$$
G_{2}=G_{1}^{*}, \quad G_{4}=G_{3}^{*}, \quad G_{6}=G_{5}^{*}, \quad G_{8}=G_{7}^{*}
$$

(здесь $G^{*}$ комплексно сопряжено с $G$ ). Базис решений уравнения (3.8) состоит из одного вектора $\vec{n}=(1,1)$, и согласно теореме 3.1 алгебра локальных симметрий уравнения Шредингера с кулоновским потенциалом $U_{Y}(s u(4, C))$ имеет вид

$$
U_{Y}(s u(4, C))=\bigoplus_{r=0}^{\infty}\left[\bigoplus_{n=0}^{r}\left(E_{1} \otimes E_{2}\right)^{n} \otimes E_{0}^{r-n}\right] .
$$

Можно непосредственно показать, что базис пространства $E_{1} \otimes E_{2}$ выражается через произведения операторов $\tilde{L}_{i j}, R_{i}$. Результат приведен в приложении. Следовательно, справедлива

ТеОрема 3.3. Все локальные симметрии уравнения Шредингера с кулоновским потенциалом принадлежат обертывающей алгебре $U(\operatorname{so}(4, C))$ алгебры so(4,C) c базисом, состоящим из генераторов $\tilde{L}_{i k}$ группы вращений в трехмерном пространстве и операторов Рунге-Ленца $R_{i}$.

Таким образом, для уравнения Шредингера для атома водорода не сушествует операторов симметрии выше второго порядка, представляюших собой аналоги векторов Рунге-Ленца.

ПРИЛОЖЕНИЕ

\section{Выражение базиса пространства $E_{1} \oplus E_{2}$ через операторы $\tilde{L}_{i j}, R_{i}$ вида (3.10)}

Обозначим

$$
\begin{aligned}
& F_{1}=\frac{1}{2}\left(L_{13}+L_{24}\right), \quad F_{2}=L_{12}-L_{34}, \quad F_{3}=X_{12}-X_{34}, \\
& F_{4}=L_{14}-L_{23}, \quad F_{5}=X_{14}-X_{23}, \quad F_{6}=\frac{1}{4}\left(X_{22}+X_{44}-X_{11}-X_{33}\right) \text {. }
\end{aligned}
$$

Здесь и ниже $L_{i j}, X_{i}$ имеют вид (2.7), $i, j=1,2,3,4 ; H, Y$ имеют вид (1.3), (3.6), соответственно.

Справедливы разложения

$$
G_{1} G_{2}=F_{1}^{2}+\frac{1}{4}\left(F_{2}^{2}+F_{4}^{2}\right)-Y^{2}+i Y
$$




$$
\begin{aligned}
G_{1} G_{4}= & \frac{1}{4}\left(F_{2} F_{3}+F_{4} F_{5}\right)+i\left(F_{6} Y-\frac{1}{4} H F_{1}\right), \\
G_{1} G_{6}= & \frac{1}{2}\left(F_{1} F_{3}-F_{4} F_{6}+\frac{1}{4} H F_{4}+F_{3} Y\right)+\frac{i}{2}\left(-F_{2} F_{6}-F_{1} F_{5}+\frac{1}{4} H F_{4}-F_{5} Y\right), \\
G_{1} G_{8}= & \frac{1}{2}\left(F_{2} F_{6}+F_{1} F_{5}+\frac{1}{4} H F_{2}-F_{5} Y\right)+\frac{i}{2}\left(F_{4} F_{6}-F_{1} F_{3}+\frac{1}{4} H F_{4}+F_{3} Y\right), \\
G_{3} G_{4}= & \frac{1}{4}\left(F_{3}^{2}+F_{5}^{2}+\omega^{2} F_{2}^{2}+\omega^{2} F_{4}^{2}\right)-\omega^{2} G_{1} G_{2}, \\
G_{3} G_{6}= & \frac{1}{2}\left(\frac{1}{4} H F_{5}+\omega^{2} F_{1} F_{2}-F_{5} F_{6}+\omega^{2} F_{2} Y\right)+ \\
& +\frac{i}{2}\left(-\omega^{2} F_{1} F_{4}+\frac{1}{4} H F_{3}-\omega^{2} F_{4} Y-F_{3} F_{6}\right), \\
G_{3} G_{8}= & \frac{1}{2}\left(F_{3} F_{6}+\omega^{2} F_{1} F_{4}+\frac{1}{4} H F_{3}-\omega^{2} F_{4} Y\right)+ \\
& +\frac{i}{2}\left(F_{5} F_{6}-\omega^{2} F_{1} F_{2}+\frac{1}{4} H F_{5}+\omega^{2} F_{2} Y\right), \\
G_{5} G_{6}= & \frac{1}{2}\left(4 F_{6}^{2}+\frac{1}{2} \omega^{2} F_{2}^{2}+\frac{1}{2} \omega^{2} F_{4}^{2}+\frac{1}{2} F_{5}^{2}+\frac{1}{2} F_{3}^{2}-4 \omega^{2} F_{1}^{2}+H F_{6}-4 \omega^{2} F_{1} Y\right)- \\
& -2 \omega^{2} G_{1} G_{2}-2 i \omega^{2} F_{1}, \\
G_{5} G_{8}= & \frac{1}{2}\left(F_{3} F_{5}-\omega^{2} F_{2} F_{4}\right)-2 \omega^{2} F_{1}-\frac{i}{2}\left(-\omega^{2} F_{2}^{2}+\omega^{2} F_{4}^{2}-F_{5}^{2}+F_{3}^{2}\right), \\
G_{7} G_{8}= & \frac{1}{2}\left(4 F_{6}^{2}+H F_{6}+4 \omega^{2} F_{1}^{2}-4 \omega^{2} F_{1} Y+\omega^{2} F_{2}^{2}+F_{5}^{2}\right)-2 \omega^{2}\left(G_{1} G_{2}-i Y\right) .
\end{aligned}
$$

С учетом соотношений (3.11) имеем

$$
\begin{array}{lll}
G_{2} G_{3}=\left(G_{1} G_{4}\right)^{*}, & G_{2} G_{5}=\left(G_{1} G_{6}\right)^{*}, & G_{2} G_{7}=\left(G_{1} G_{8}\right)^{*}, \\
G_{4} G_{5}=\left(G_{3} G_{6}\right)^{*}, & G_{4} G_{7}=\left(G_{3} G_{8}\right)^{*}, & G_{6} G_{7}=\left(G_{5} G_{8}\right)^{*} .
\end{array}
$$

Редукция уравнения (1.3) к уравнению (1.5) (следует положить $Y=0$ ) приводит операторы (П.1) к виду

$$
\begin{array}{ll}
F_{1}=\tilde{L}_{12}, & F_{2}=-2 \tilde{L}_{13}, \quad F_{4}=-2 \tilde{L}_{23} ; \\
F_{4}=-4 R_{1}, & F_{5}=-4 R_{2}, \quad F_{6}=2 R_{3},
\end{array}
$$

где $\tilde{L}_{i j}, R_{i}$ имеют вид (3.10), оператор $H$ принимает вид (1.5). В результате базисные элементы пространства $E_{1} \otimes E_{2}$ выражаются через произведения операторов $\tilde{L}_{i j}, R_{i}$ и оператор $H$.

\section{Список литературы}

[1] Овсянников Л.В. Груповой анализ дифференциальных уравнений. М.: Наука, 1978.

[2] Ибрагимов H.X. Группы преобразований в математической физике. М.: Наука, 1983.

[3] Олвер П. Приложения групп Ли к дифференциальным уравнениям. М.: Мир, 1989.

[4] Фущич В.И., Никитин А.Г. Симметрия уравнений квантовой механики. М.: Наука, 1990. 
[5] Шаповалов В.Н. // Дифф. уравнения. 1980. Т. 16. №10. С. 1864-1874.

[6] Миллер У. Симметрия и разделение переменных. М.: Мир, 1981.

[7] Шаповалов А.В., Широков И.В. // Изв. вузов. Физика. 1991. № 4. С. 95-100.

[8] Никитин А.Г., Прилипко А.И. Симметрия и решения уравнений математической физики. Киев: Ин-т математики Украины, 1989. С. 40-44.

[9] Мешков А.Г. // ТМФ. 1983. Т. 55. № 2. С. 197-204.

[10] Шаповалов А.В., Широков И.В. // ТМФ. 1992. Т. 92. №1. С. 3-12.

[11] Шаповалов В.Н. // Изв. вузов. Физика. 1977. №6. С. 57-70.

[12] Фок В.А. // Изв АН СССР (отд. матем. и естеств. наук). 1935. № 2. С. 169-179.

[13] Bargman V. // Z. Physik. 1936. Bd. 99. S. 576.

[14] Barut A.O., Bohm A. // Phys.Rev. B. 1965. V. 15. P. 1107-1112.

[15] Dothan Y., Gell-Mann M., Ne'eman Y. // Phys. Lett. 1965. V. 15. P. 148-151.

[16] Mukunda N., O'Raifeartaigh L., Sudarshan E. // Phys. Rev. Lett. 1965. V. 15. P. 1041-1044.

[17] Малкин И.А., Манько В.И. // Письма в ЖЭЭФ. 1965. Т. 2. С. 230-234.

[18] Малкин И.А., Манько В.И. // ЯФ. 1966. Т. 3. В. 2. С. 372-382.

[19] Манько В.И. Симплектическая группа и инварианты квантовых систем / Матем. анализ. (Итоги науки и техники.). М.: ВИНИТИ, 1984. Т. 22. С. 59-100.

[20] Додонов В.В., Манько В.И. // Тр. ФИАН СССР им. П.Н. Лебедева. 1987. Т. 183. С. 71-181.

[21] Малкин И.А., Манько В.И. Динамические симметрии и когерентные состояния квантовых систем. М.: Наука, 1979.

[22] Барут A., Рончка Р. Теория представлений групп и ее приложения. Т. 1, 2. М.: Мир, 1980.

[23] Xapm H. Геометрическое квантование в действии. М.: Мир, 1985.

[24] Брычков Ю.А., Прудников А.П. Интегральные преобразования обобщенных функций. М.: Наука, 1977.

Томский государственный университет Омский государственный университет

\section{A. A. Drokin, A. V. Shapovalov, I. V. Shirokov \\ LOCAL SYMMETRY ALGEBRA OF SHRÖDINGER EQUATION FOR HYDROGEN ATOM}

The complete description of local symmetries (which are differential operators of arbitrary finite order) is given for stationary Shrödinger equation for Hydrogen atom. This is done using the reduction of Shrödinger equation for isotropic harmonic oscillator to one for the Hydrogen atom, which induces the correspondent symmetry algebras reduction. It is shown that all nontrivial local symmetry operators for $n$-dimensional isotropic harmonic oscillator belong to enveloping algebra $U(s u(n, C))$ of algebra $s u(n, C)$. For Hydrogen atom all nontrivial local symmetries constitute enveloping algebra $U(s o(4, C))$ of algebra $s o(4, C)$. Basis of $s o(4, C)$ consists of rotation group generators and Runge-Lenz-operators. 